\title{
Expression and prognostic significance of Golgiglycoprotein73 (GP73) with Epithelial- mesenchymal transition (EMT) related molecules in Hepatocellular Carcinoma (HCC)
}

Yong xing Bao, Qian $\mathrm{Cao}^{\dagger}$, Ying Yang ${ }^{\dagger}$, Rui Mao, Lei Xiao, Hua Zhang, Hua-rong Zhao and Hao Wen

\begin{abstract}
Background: Hepatocellular carcinoma (HCC) is the fifth most common cancer and the third cause of cancer-related deaths, worldwide. It is essential to develop an effective prognostic biomarker and determine the mechanisms underlying HCC invasion and metastasis.

Aims: This study aimed to investigate the expression of Golgi glycoprotein73 (GP73) and Epithelial-mesenchymal transition (EMT) molecules such as E-cadherin and Vimentin in HCC. We also evaluated the prognostic value of GP73 in HCC.

Methods: Immunohistochemistry (IHC) was used to determine the expression of GP73 and EMT molecules in 75 HCC specimens and the corresponding paracarcinomatous liver (PCL) tissues. Spearman's correlation test was used to analyze the correlation of GP73 and EMT molecules. Clinicopathological features of the HCC patients were also analyzed. Univariate survival analysis was performed by the Kaplan-Meier method and differences among the groups were analyzed by the Log-rank test.

Results: GP73 expression in HCC was higher compared with PCL tissues $\left(X^{2}=73.60, P<0.05\right)$. EMT molecules were also detected in HCC and PCL tissues. GP73 was negatively correlated with E-cadherin ( $r=-0.49, P<0.05)$, but positively correlated with Vimentin $(r=0.46, P<0.05)$ in HCC. GP73 was correlated with the clinicopathological features including Edmondson grade, vascular invasion and TNM stage $(P<0.05)$, which was also associated with overall survival $(O S)(P<0.05)$.

Conclusions: GP73 was negatively with E-cadherin and positively correlated with Vimentin. It might be associated with aggressive behavior of HCC and had influence on patients' OS. Further research is needed to determine the potential of GP73.

Virtual slides: The virtual slide(s) for this article can be found here: http:/www.diagnosticpathology.diagnomx.eu/29 vs/ 1504046946108618; http://med.motic.com/MoticGallery/Slide?id=3b6a037e-f60e-4c68-9106-41e790de9431\&user=2C69F0D6A478-4A2B-ABFO-BB36763E8025; http://med.motic.com/MoticGallery/Slide?id=a25b5b32-b613-47b0-9f8bele67a95d1 bf\&user=2C69F0D6-A478-4A2B-ABF0-BB36763E8025.
\end{abstract}

\section{Background}

Hepatocellular carcinoma (HCC) accounts for most liver cancers worldwide, with poor prognosis [1]. The poor prognosis is attributed to extensive regional invasion and distant metastasis during the initial diagnosis. However, the mechanism underlying local invasion and distant

\footnotetext{
*Correspondence: wenhao@126.com

${ }^{\dagger}$ Equal contributors

Tumor Department, First Affiliated Hospital of Xinjiang Medical University (XJMU), Urumqi, China
}

metastasis are still unclear. Therefore, the need for effective molecular markers to evaluate the prognosis of $\mathrm{HCC}$ cannot be overstated.

Recently, a new biomarker Golgi glycoprotein73 (GP73) has been investigated for its diagnostic accuracy and potential clinical application in HCC. GP73 was first discovered in 2000 [2]. Many reports suggest that the GP73 expression was significantly increased in HCC unlike normal human liver cells [3]. The sensitivity and specificity of GP73 were higher than AFP, making it an ideal marker for 
early diagnosis of HCC $[2,4,5]$. However, research studies of GP73 until now have focused merely on its role in early diagnosis. Reports investigating the role of GP73 in clinical pathology and patients' OS are rare.

Epithelial-mesenchymal transition (EMT) is a useful prognostic marker for survival in patients. EMT has a close relationship with tumor invasiveness and metastasis. The most representative molecules are E-cadherin and Vimentin [6-8].

Therefore, we explored the expression of GP73, E-cadherin and Vimentin in HCC /PCL tissues. The aim of the study was to find the relationships among GP73 and EMT molecules and to evaluate the role of GP73 in predicting the prognosis in $\mathrm{HCC}$.

\section{Methods}

\section{Patients and tissue samples}

A total of 75 samples, including HCC and PCL tissues were obtained by surgical resection from the First Affiliated
Hospital of Xinjiang Medical University (XJMU), between 2007 and 2012. Insufficient liver tissue in the biopsy specimen or insufficient clinical data regarding patient outcomes, were exclusion criteria. None of the patients received chemotherapy or radiotherapy prior to surgery. All patients were followed-up via telephone or questionnaires. Survival was calculated from tissue diagnosis until the patient's death or termination of follow-up (April 2013). We obtained consent from all patients, and determined the clinic pathology including gender, age, AFP, HBV infection, thrombosis, tumor differentiation, vascular invasion and TNM stage. The TNM classification system was in accordance with the American Joint Committee on Cancer/International Union Against Cancer (AJCC/ UICC). The tumor differentiation was based on the Edmondson grading.

The tissue specimens were resected from the HCC patients. We obtained the consent of all patients. Research carried out on patients was in compliance with
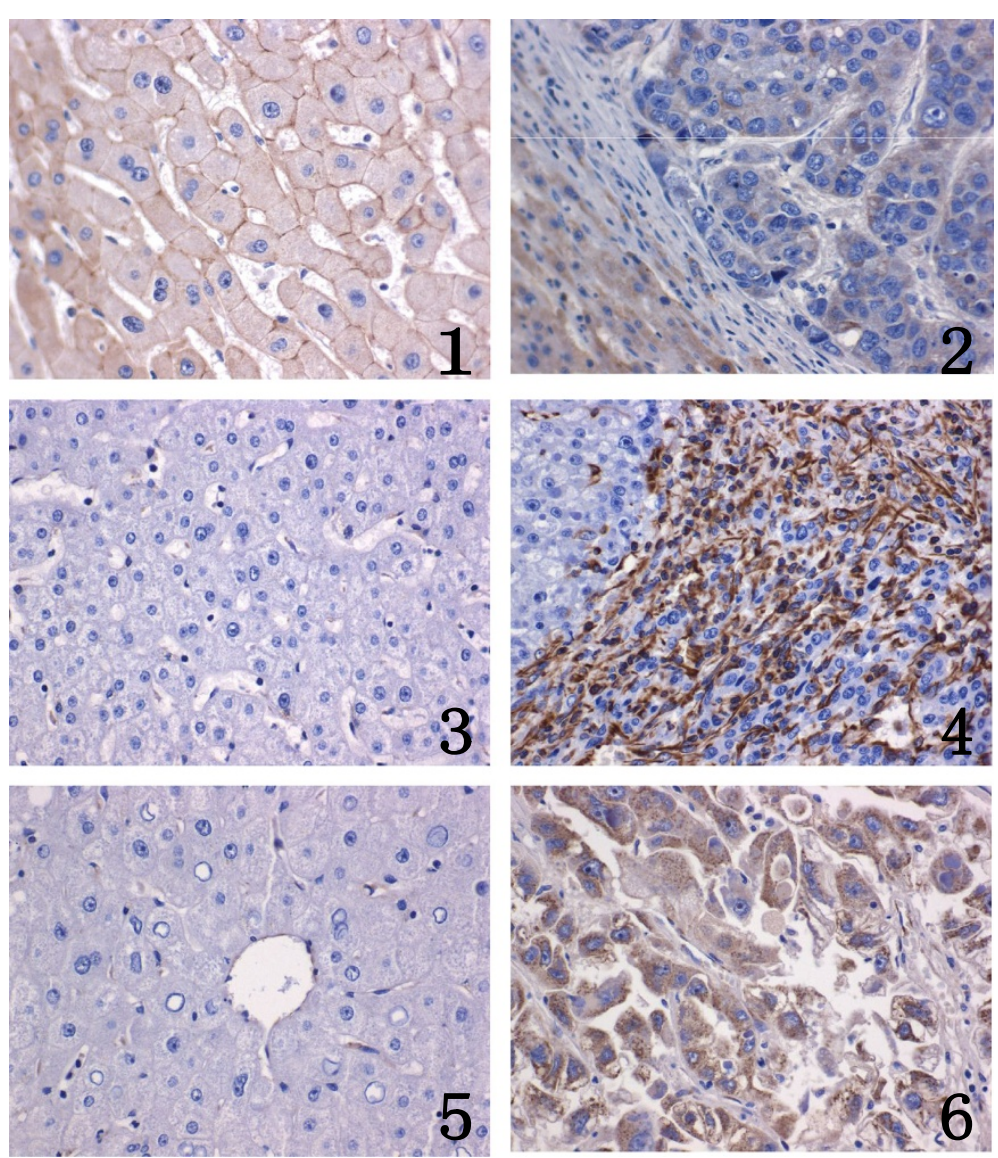

Figure 1 Immunohistochemistry of E-cadherin, Vimentin and GP73 in PCL tissues and HCC tissue (400x). E-cadherin was detected in the cell membrane. Note that E-cadherin showed moderate and strong staining in S (PCL) (1) By contrast, it was almost absent in normal control HCC (2) (×400).Vimentin was detected in the cytoplasm and interstitial cells. Note that Vimentin was almost absent in normal controls (PCL) (3) By contrast, moderate and strong staining was seen in HCC (4) (×400).GP73 protein expression was evaluated by an En-Vision immunohistochemical method. Note that GP73 was almost absent in normal controls (PCL) (5). By contrast, moderate and strong staining of GP73 was identified in HCC, respectively. (×400). (6). 
Table 1 GP73, E-cadherin and Vimentin in HCC and the PCL tissues

\begin{tabular}{lllllll}
\hline Expression & $\mathbf{N}$ & Positive & Negative & Positive rate & $\boldsymbol{X}^{2}$ & $\boldsymbol{P}$ \\
\hline GP73 & & & & & & \\
HCC & 75 & 54 & 21 & $72.00 \%$ & & \\
PCL & 75 & 3 & 72 & $4.00 \%$ & 73.60 & $<0.05$ \\
E-cadherin & & & & & & \\
HCC & 75 & 18 & 57 & $24.00 \%$ & & \\
PCL & 75 & 66 & 9 & $88.00 \%$ & 62.34 & $<0.05$ \\
Vimentin & & & & & & \\
HCC & 75 & 45 & 30 & $60.00 \%$ & & \\
PCL & 75 & 9 & 66 & $12.00 \%$ & 37.50 & $<0.05$ \\
\hline
\end{tabular}

the Helsinki Declaration and approved by the Xinjiang Medical Ethics Committee.

\section{Reagents}

Rabbit anti-human GP73 polyclonal antibody was purchased from American Proteintech Group. Rabbit anti-

Table 2 The correlation between GP73 and clinic pathologic features

\begin{tabular}{|c|c|c|c|c|c|c|}
\hline Variable & $\mathbf{N}$ & Positive & Negative & $\begin{array}{l}\text { Positive } \\
\text { rate }\end{array}$ & $x^{2}$ & $P$ \\
\hline \multicolumn{7}{|l|}{ Gender } \\
\hline Male & 51 & 39 & 12 & $76.47 \%$ & & \\
\hline Female & 24 & 15 & 9 & $62.50 \%$ & 1.58 & 0.21 \\
\hline \multicolumn{7}{|l|}{ Age } \\
\hline$>60 y$ & 33 & 27 & 6 & $81.82 \%$ & & \\
\hline$\leq 60 y$ & 42 & 27 & 15 & $64.29 \%$ & 2.82 & 0.09 \\
\hline \multicolumn{7}{|l|}{$\mathrm{HBsAg}$} \\
\hline Positive & 27 & 6 & 21 & $77.78 \%$ & & \\
\hline Negative & 48 & 15 & 33 & $68.75 \%$ & 0.70 & 0.40 \\
\hline \multicolumn{7}{|l|}{ AFP value } \\
\hline$>400 \mathrm{ng} / \mathrm{ml}$ & 12 & 9 & 3 & $75.00 \%$ & & \\
\hline$\leq 400 \mathrm{ng} / \mathrm{ml}$ & 63 & 45 & 18 & $71.43 \%$ & 0.06 & 0.80 \\
\hline \multicolumn{7}{|l|}{ Thrombosis } \\
\hline Yes & 30 & 21 & 9 & $70.00 \%$ & & \\
\hline No & 45 & 33 & 12 & $73.33 \%$ & 0.10 & 0.75 \\
\hline \multicolumn{7}{|c|}{ Edmondson grade } \\
\hline$|-| \mid$ & 15 & 3 & 12 & $20.00 \%$ & & \\
\hline III-IV & 60 & 51 & 9 & $85.00 \%$ & 25.15 & $<0.05$ \\
\hline \multicolumn{7}{|c|}{ Vascular invasion } \\
\hline Yes & 36 & 18 & 18 & $50.00 \%$ & & \\
\hline No & 39 & 36 & 3 & $92.31 \%$ & 16.62 & $<0.05$ \\
\hline \multicolumn{7}{|l|}{ TNM stage } \\
\hline $\mathrm{T} 1 / 2$ & 21 & 9 & 12 & $42.86 \%$ & & \\
\hline Т 3/4 & 54 & 45 & 9 & $83.33 \%$ & 12.29 & $<0.05$ \\
\hline
\end{tabular}

human E-cadherin polyclonal antibodies and mouse anti-human Vimentin monoclonal antibody were purchased from Booster Company. Immunohistochemistry kit antibodies were purchased from Zhongshan Jinqiao Biotechnology Development Corporation.

\section{Major instruments}

Microscope, microwave oven and medical clean bench were offered by the First Affiliated Hospital of XJMU.

\section{Immunohistochemistry (IHC)}

Immunohistochemistry was performed using En-Vision method for IHC staining. We fixed the specimens in $10 \%$ neutral formalin and used paraffin to embed them. We obtained deparaffinized 5- $\mu \mathrm{m}$ thicksections from the FFPE tissue blocks with xylene, followed by rehydration using a graduated series of ethanol. We used $3 \% \mathrm{H}_{2} \mathrm{O}_{2}$ to block endogenous peroxides. Microwave oven was used to block non-specific antibody binding. The sections were incubated separately overnight at $4^{\circ} \mathrm{C}$ with the primary antibodies, at a concentration of 1:50. The secondary antibodies were then added to the specimens the next day. $\mathrm{DAB}$ was used as the chromogen. The staining was terminated with water and the specimens counterstained with haematoxylin.

\section{Evaluation of IHC staining results}

IHC staining results were interpreted independently by two pathologists blinded to the outcomes. An intensity score was assigned, representing the average intensity of positive cells ( 0 , none; 1 , weak; 2 , intermediate; 3 , strong). A proportion score was assigned, which represented the estimated proportion of positive-staining cells ( 0 , no positive cells; $1,0-20 \% ; 2,21-50 \% ; 3,51-80 \%$; 4, 81-100\%). The proportion and intensity scores were multiplied with a immunoreactivity score (IS). The IS was further divided as follows: $0-1(-)$; 2-4 (+) ; 5-7 (++); >8(+++). “- ++" was regard as low level," $++\sim+++$ " was defined as high level.

\section{Statistics}

The appropriate non-parametric tests were used to investigate GP73 expression and clinicpathological parameters. Unvaried survival analysis was performed by the KaplanMeier method, and differences between the groups were analyzed by the log-rank test. Two-tailed p-values of $<0.05$ were considered significant. All statistical analyses were performed using SPSS software version 17.0 (SPSS for Windows, Chicago, IL, USA).

\section{Results}

The expression of GP73 and EMT molecules in HCC and $\mathrm{PCL}$ tissues and the inter-relationships

GP73 was detected in the cytoplasm of liver cells, which is brown. E-cadherin was detected as a membrane-linear/ 


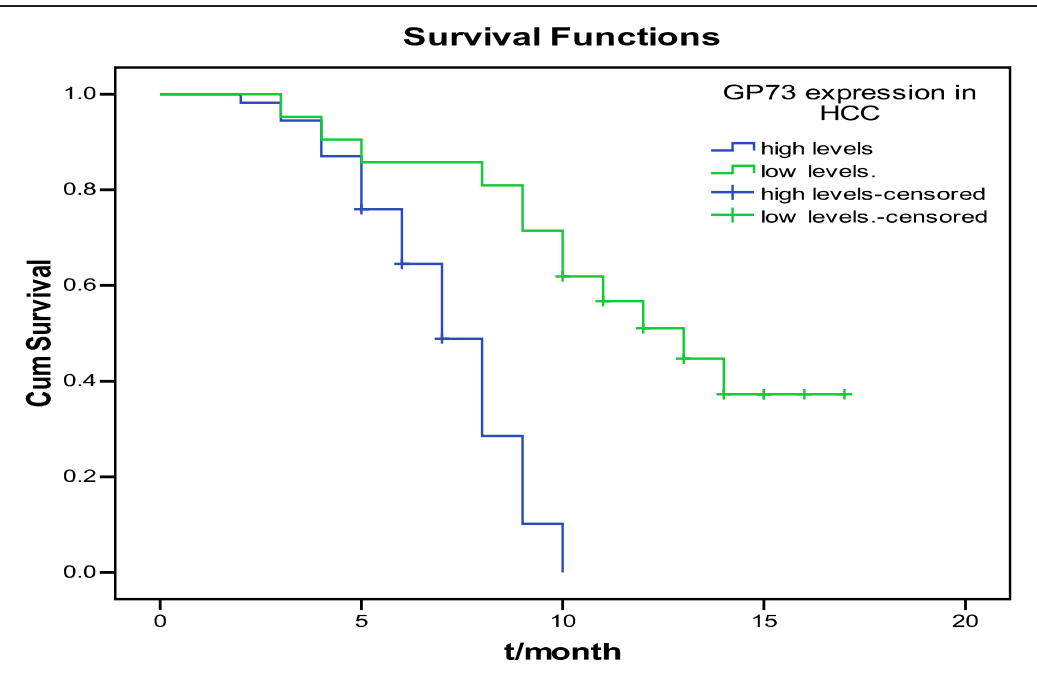

Figure 2 Survival curves of HCC patients with different levels of GP73 expression. The surival curves showed that HCC patients with high levels of GP73 expression were different from patients with low levels.

disarrayed immunostaining pattern, while Vimentin labeling was observed in cytoplasmic and interstitial cells (Figure 1). Data showed that GP73 staining was detected up to $72 \%(54 / 75)$ in $\mathrm{HCC}$, but only up to $4 \%(3 / 75)$ in the PCL tissues. GP73 expressed in HCC was higher compared with PCL $\left(\chi^{2}=73.60, P<0.05\right)$. The expression of E-cadherin in HCC was $24 \%$ (18/75) compared with PCL tissues $88 \%(66 / 75)$. The increased expression of Vimentin in HCC $[60 \%(45 / 75)]$ was higher than in PCL tissues [12\% (9/75)] (Table 1).

\section{Correlation between GP73 and clinical pathology}

GP73 was associated with Edmondson grade, vascular invasion and TNM stage. $(P<0.05)$. No correlation was detected between GP73 with gender, age, HBV infection, AFP, or thrombosis $(P>0.05)$ (Table 2$)$.

\section{GP73 expression with survival in HCC patients}

We divided the 75 specimens into high and low levels. Kaplan-Meier survival analysis and Log-rank test showed that GP73 expression was significantly associated with the OS (Figure 2, Table 3). The median survival time of patients with high levels of GP73 was 7 months compared with 13 months associated with low GP73.

Table 3 Kaplan-Meier survival analysis and log-rank test of GP73

\begin{tabular}{llllll}
\hline GP73 & N & Median OS/month & $\mathbf{9 5 \%}$ Cl & $\boldsymbol{X}^{2}$ & $\boldsymbol{P}$ \\
\hline High levels & 54 & 7.00 & $6.25 \sim 7.75$ & 28.18 & $<0.05$ \\
Low levels & 21 & 13.00 & $9.27 \sim 16.73$ & & \\
\hline
\end{tabular}

\section{Discussion}

$\mathrm{HCC}$ is the most common primary liver cancer [9]. It is the third leading cause of cancer-related death preceded only by lung and stomach cancers [10]. Although patients with early diagnosis have benefited from advances in treatment, the prognosis in $\mathrm{HCC}$ is still disappointing [11], largely due to the highly invasive and metastatic potential [12]. Therefore, it is essential to find an effective biomarker for HCC prognosis and underlying mechanisms of invasion and metastasis.

GP73 is a $73 \mathrm{kDa}$ type-II Golgi transmembrane glycoprotein, which was originally cloned from a library derived from patients with adult giant-cell hepatitis [2]. Research suggested that GP73 was overexpressed in HCC [3]. Mao et al reported in a large sample of international groups, that the sensitivity and specificity of GP73 in HCC were higher than AFP. It suggested that GP73 was a new type of molecular marker in early diagnosis of HCC [4]. Our data showed that tissue GP73 levels were higher level in HCC compared with PCL tissues, consistent with the preliminary studies.

In 1982, Garry Greenburg and Hay first proposed EMT [13]. Accumulating evidence indicated that EMT was a pivotal mechanism contributing to cancer invasion and metastasis. EMT involves multiple components, such as E-cadherin and Vimentin.

E-cadherin expression is a hallmark of EMT [14]. E-cadherin expression is associated with the establishment of cell polarity and tissue organization. Reduced E-cadherin expression contributes to the transition of adenoma to carcinoma in animal models and is inversely correlated with tumor stage [15-17]. Vimentin is a component of type III intermediate filaments and the archetypal mesenchymal marker most commonly used to categorize EMT. 
Vimentin expression is a late event in EMT, preceded by a loss of epithelial features and leading to upregulation of mesenchymal genes [18]. Huang found that Vimentin expression was significantly higher in the metastasis group than non-metastasis group $(P \leq 0.05)$ unlike E-cadherin [19].

In the present study, IHC analysis of the expression of E-cadherin, Vimentin and GP73 showed that GP73 and Vimentin levels were higher in HCC compared with PCL tissues, unlike E-cadherin. Spearman's correlation test indicated that GP73 was positively associated with Vimentin, but negatively with E-cadherin. The results resembled the findings of Huang et al.

Several studies have demonstrated that GP73 overexpression may be associated with tumor invasiveness $[1,4,5,7,20]$. Sun reported that the level of GP73 was strongly associated with tumor size, vein invasion, and tumor differentiation, which suggested that GP73 augmented tumor invasion and metastasis [20]. Our research found that GP73 was significantly correlated with the Edmondson grade, vascular invasion and TNM stage (all $P<0.05$ ) (Table 2). These clinicopathological features are characteristic of tumor invasiveness and metastatic potential. In conjunction with the relationships among GP73 and EMT, we hypothesize that GP73 might be associated with invasive behavior in HCC.

In this study, survival analysis showed that patients with high level of GP73 showed poorer OS compared with low levels. We presumed that GP73 might correlate with patients' prognosis, contrary to Sun et al. Notably, Sun proved that serum GP73 has no correlation with prognosis, but the correlation of tissue GP73 and prognosis are unknown. There exist a differentiation between serum and tissue GP73, more studies are needed to prove the prognostic value of GP73. And the results may be affected by the number of specimens, histological type and pathological grading method [21], different results were permitted.

Our study limitations relate to the small sample size of the patients' cohort with only 75 patients. The group size became even smaller when divided by the high level and low level of GP73 expression. Nonetheless, multiple logistic regression analysis was applied to assess parameters independently associated with GP73.

\section{Conclusions}

In summary, GP73 expression positively correlated with EMT molecules. High level of GP73 was associated with Edmondson grade, vascular invasion and TNM stage. Therefore, we conclude that GP73 involved in the regulation of EMT to obtain invasion on HCC and it would be a biomarker for assessing the prognostic values. GP73 is a prognostic biomarker. However, The regulation of GP73 and its function are controversial and have yet to be clarified [22]. How its occurrence in the HCC still needs to be elucidated.

\section{Competing interests}

We certify that we have participated sufficiently in the work to take public responsibility for the appropriateness of the experimental design and method, and the collection, analysis, and interpretation of the data. We have reviewed the final version of the manuscript and approve it for publication. To the best of our knowledge and belief, this manuscript has not been published in whole or in part nor is it being considered for publication elsewhere.

\section{Authors' contributions}

$Y-X B, Q C$ and $Y Y$ have made substantial contributions to conception and design, and acquisition and analysis of data. RM and LX have been involved in drafting the manuscript or revising it critically for important intellectual content. Y-XB has given final approval of the version to be published. All authors read and approved the final manuscript.

\section{Acknowledgements}

This work was encouraged by the members of the Tumor Department of Hospital of Xinjiang Medical University. We thank the Pathology Department of the First Affiliated Hospital of Xinjiang Medical University for providing us with the specimens. We also thank the American Proteintech Group company and Booster Company, which offered the antibody.

Received: 19 September 2013 Accepted: 15 November 2013 Published: 6 December 2013

\section{References}

1. Yang Y, Xiao L, Bao Y, et al: Diagnostic value of Golgi-73 and AFP alone or combination in primary hepatocelluar carcinoma. Chin J Lab Med 2012, 35(11):1034-1037.

2. Kladney RD, Bulla GA, Guo L, et al: GP73, a novel Golgi-localized protein upregulated by viral infection. Gene 2000, 249(1-2):53-65.

3. Parkin DM: Global cancer statistics in the year 2000. Lancet Oncol 2001, 2:533-543.

4. Mao $\mathrm{Y}$, Yang $\mathrm{H}, \mathrm{Xu} \mathrm{H}$, et al: Golgi protein $73(\mathrm{GOLPH} 2)$ is a valuable serum marker for hepatocellular carcinoma. Gut 2010, 59:1687-1693.

5. YangY XL, Bao Y: Expression profiles and differential diagnostic value of serum Golgi protein-73 in patients with liver cirrhosis and primary hepatic carcinoma. Chin J Hepatol 2012, 20(12):920-924.

6. Mima K, Okabe H, Ishimoto T, et al: CD44s regulates the TGF- $\beta$-mediated mesenchymal phenotype and is associated with poor prognosis in patients with hepatocellular carcinoma. Cancer Res 2012, 72:3414-3423.

7. Yongli S, Hongyan W, Mingyi Z, et al: Expression of the epithelialmesenchymal transition-related proteins and their clinical significance in lung adenocarcinoma. Diagn Pathol 2013, 8:89.

8. Kosuke M, Hiromtsu H, et al: Epithelial-mesenchymal transition expression profiles as a prognostic factor for disease-free survival in hepatocellular carcinoma: clinical significance of transforming growth factor-signaling. Oncol Lett 2013, 5:149-154

9. Nehal AR, Nagla SA: The diagnostic value of arginase-1 immunostaining in differentiating hepatocellular carcinoma from metastatic carcinoma and cholangiocarcinoma as compared to HepPar-1 Radwan and Ahmed. Diagn Pathol 2012, 7:149.

10. Waly RS, Yangde Z, Yuxiang C: Hepatocellular carcinoma: focus on different aspects of management. Oncology 2012, 42:1673.

11. Jemal A, Bray F, Center MM, et al: Global cancer statistics. Clin 2011, 61(2):69-90

12. Murray G, Duncan M, Arbuckle $E$, et al: Matrix metalloproteinases and their inhibitors in gastric cancer. Gut 1998, 43:791-797.

13. Yang J, Mani SA, Donaher JL, et al: Twist, master regulator of morphogenesis, plays an essential role in tumor metastasis. Cell 2004 117(5):927-939.

14. Shen $\mathrm{R}$, Xie F, Shen $\mathrm{H}$, et al: Negative correlation of LIV-1 and E-cadherin expression in hepatocellular carcinoma cells. PLoS One 2013, 8:2.

15. Shiozaki $\mathrm{H}, \mathrm{Oka} H$, Inoue $\mathrm{M}$, et al: E-cadherin mediated adhesion system in cancer cells. Cancer 1996, 77:1605-1613. 
16. Bukholm IK, Nesland JM, Karesen R, et al: E-cadherin and a-, b-, and c-catenin protein expression in relation to metastasis in human breast carcinoma. Pathology 1998, 185:262-266.

17. Van AE, De WO, Correia AS, et al: Defective Ecadherin/catenin complexes in human cancer. Virchows Arch 2001, 439:725-751.

18. Kokkinos MA, Wafai $R$, Wong MK, et al: Vimentin and epithelialmesenchymal transition in human breast cancer-observations in vitro and in vivo. Cells Tissues Organs 2007, 185:191-203.

19. Qiang H, Lei L, Chen HL, et al: Expression of Smad7 in Cholangiocarcinoma: prognostic Significance and Implications for Tumor Metastasis. Asian Pac J Cancer Prev 2012, 13:125-129.

20. Sun $Y$, Yang $H$, Mao $Y$, et al: Increased Golgi protein 73 expression in hepatocellular carcinoma tissue correlates with tumor aggression but not survival. Hepatology 2011, 26(7):1207-1212.

21. Leong AS: Diagnostic immunohistochemistry problems and solutions. Pathology 1992, 24:1.

22. Lorinda MW, Sheri Y, Maria MP: Decreased survival and hepatorenal pathology in mice with C-terminally truncated GP73. Clin Exp Pathol 2009, 2:34-47.

doi:10.1186/1746-1596-8-197

Cite this article as: Bao et al.: Expression and prognostic significance of Golgiglycoprotein73 (GP73) with Epithelial-mesenchymal transition (EMT) related molecules in Hepatocellular Carcinoma (HCC). Diagnostic Pathology 2013 8:197.

\section{Submit your next manuscript to BioMed Central and take full advantage of:}

- Convenient online submission

- Thorough peer review

- No space constraints or color figure charges

- Immediate publication on acceptance

- Inclusion in PubMed, CAS, Scopus and Google Scholar

- Research which is freely available for redistribution 